\title{
Factores que influyen en la decisión de utilizar anestesia epidural en las mujeres
}

\section{Factors influencing the decision to use epidural anesthesia for women}

\section{Fatores que influenciam a decisão de usar anestesia peridural para mulheres}

\author{
María Josefa Campoy Quesada ${ }^{1}$ y Juan Pedro De Haro García ${ }^{2}$ \\ ${ }^{1}$ Diplomada Universitaria en Enfermería en Hospital Comarcal del Noroeste de la Región de \\ Murcia y Máster en Salud, Mujer y Cuidados. \\ ${ }^{2}$ Diplomado Universitario en Enfermería en Hospital Virgen de la Arrixaca de Murcia \\ Cómo citar este artículo en edición digital: Campoy Quesada, Ma.J. y De Haro García, J.P. (2014) Factores que influyen en la deci- \\ sión de utilizar anestesia epidural en las mujeres. Cultura de los Cuidados (Edición digital) 18, 39. \\ Disponible en: http://dx.doi.org/10.7184/cuid.2014.39.14> \\ Correspondencia; C/ Calderón de la Barca, No32, Bullas (Murcia) C.P. 30180 \\ Correo electronico: marijoseck@hotmail.com \\ Recibido: 01/12/2013; Aceptado: 14/04/2014
}

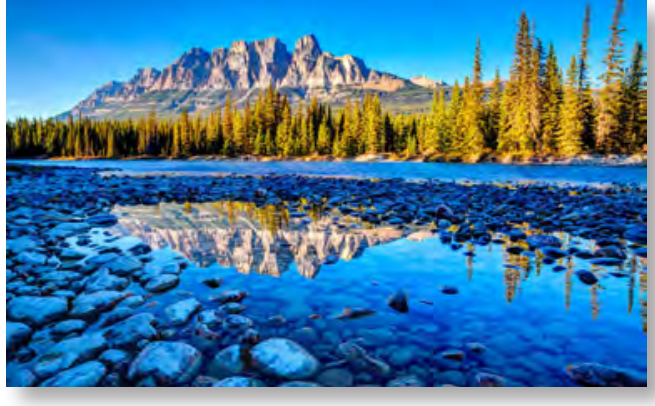

ABSTRACT

Justification: Immigration in Spain is a very important phenomenon which reverberates in the field of the health.

Objective: To investigate the relation between the choice of epidural anesthesia and the nationality of the women, and in turn, to observe if other factors also influence this decision.

Design and Methodology: Quantitative, cross-sectional and descriptive study of 634 women who have given birth at the Northwestern Hospital in the Region of Murcia. The variables selected were: obstetric history, age, type of anesthesia, the nationality of women and reasons of rejection of epidural anesthesia.
Results: The 8,51\% of women who have given birth in the Northwestern Hospital in the Region of Murcia in 2010 were immigrants. As for nationality, 20, 37\% of women immigrants have not used any type of anesthesia. We found a significant difference with the Spanish immigrant women in which only $4,31 \%$ rejected $(\mathrm{P}<0,001)$. In relation to abortions, women who do not prefer any anesthesia are those who do have abortions $(\mathrm{P}<0,05)$. Younger ages for women, 16 to 25 years, is associated with no use of epidural anesthesia $(\mathrm{P}<0,05)$.

Conclusion: Immigrant women make less use of epidural anesthesia. It is a challenge for nurses to learn the different cultures and to carry out work in an increasingly multicultural society.

Keywords: immigrants, epidural anesthesia, transcultural nursing.

\section{RESUMO}

Justificação: Imigração na Espanha é um fenômeno muito importante que afeta a área da saúde. 
Objetivo: Investigar a relação entre a escolha de anestesia peridural ea nacionalidade da mulher, e por sua vez, para ver se outros fatores também influenciam esta decisão.

Projeto e Metodologia: quantitativa, o estudo descritivo transversal de uma amostra de 634 mulheres que deram à luz no Hospital da Região Noroeste de Múrcia. As variáveis selecionadas foram: história obstétrica, idade, tipo de anestesia, as mulheres nacionais e motivos da recusa da anestesia epidural.

Resultados: 8,51 \% das mulheres que deram à luz no hospital da Região Noroeste de Murcia em 2010 eram imigrantes. Em relação a nacionalidade, 20,37\% das mulheres imigrantes não usei nenhuma anestesia. Encontramos uma diferença significativa com nenhuma mulher imigrante espanhol em que apenas 4,31\% rejeitados $(\mathrm{P}<0,001)$. No que diz respeito ao aborto, as mulheres que não preferem qualquer anestesia são aqueles que têm abortos ( $P$ $<0,05)$. Jovens mulheres com idades entre $16 \mathrm{a}$ 25 , não estava relacionado com o uso de anestesia epidural $(\mathrm{P}<0,05)$.

Conclusão: As mulheres imigrantes fazem menos uso de anestesia epidural. É um desafio para a enfermeira aprender diferentes culturas e obter o seu trabalho em uma sociedade cada vez mais multicultural.

Palavras-chave: imigrantes, a anestesia epidural, enfermagem transcultural.

\section{RESUMEN}

Justificación: La inmigración en España es un fenómeno de gran importancia que repercute en el área de la salud.

Objetivo: Investigar la relación entre la elección de la anestesia epidural y la nacionalidad de la mujer, y a su vez, observar si influyen además otros factores en esta decisión.

Diseño y Metodología: Estudio cuantitati- vo, transversal y descriptivo con una muestra de 634 mujeres que han dado a luz en el Hospital del Noroeste de la Región de Murcia. Las variables seleccionadas fueron: antecedentes obstétricos, edad, tipo de anestesia, nacionalidad de la mujer y motivo por el rechazo de la anestesia epidural.

Resultados: El 8,51\% de las mujeres que dieron a luz en el hospital del Noroeste de la Región de Murcia durante el año 2010 fueron inmigrantes. En cuanto a la nacionalidad, el $20,37 \%$ de las mujeres inmigrantes no han utilizado ningún tipo de anestesia. Se ha encontrado una significante diferencia con las mujeres no inmigrantes españolas en las que sólo el $4,31 \%$ la rechazó $(\mathrm{P}<0,001)$. En relación a los abortos, las mujeres que no prefieren ningún tipo de anestesia son aquellas que sí han tenido abortos $(\mathrm{P}<0.05)$. Las edades más jóvenes de las mujeres, de 16 a 25 años, se relaciona con el no uso de la anestesia epidural $(\mathrm{P}<0,05)$.

Conclusión: Las mujeres inmigrantes hacen menos uso de la anestesia epidural. Es un reto para la enfermera aprender las diferentes culturas ya que realiza su trabajo en una sociedad cada vez más multicultural.

Palabras clave: inmigrantes, anestesia epidural, enfermería transcultural.

\section{INTRODUCCIÓN}

La migración es un tema cada vez más importante en nuestro país, ya que la situación geográfica y económica de España la convierte en uno de los principales destinos de los movimientos migratorios. Las comunidades con mayor porcentaje de extranjeros son Cataluña (866.800 y $12,2 \%)$, Madrid (695.600 y $11,8 \%$ ), Comunidad Valenciana (641.000 y 13,4\%), Región de Murcia (181.800 y 13,3\%) e Islas Baleares (153.700 y 15,6\%). (Fernández, 2006) 
Actualmente, la inmigración en nuestro país ha aumentado de un modo considerable constituyendo un fenómeno muy importante, el cual repercute también en el área de la salud. Por lo tanto, es fundamental conocer sus características y así poder ofrecer una asistencia más adecuada y adaptada a sus necesidades, como es el mejor seguimiento y una mejor atención a las madres gestantes y a sus recién nacidos. Entre el 60 y $80 \%$ de las mujeres que emigran hacía nuestro país tienen edades comprendidas entre los 20 y los 40 años, es decir, en plena edad fértil, lo cual queda de manifiesto por el notable aumento de nacimientos procedentes de madres extranjeras que se está produciendo en España. (Manzanares, 2008). Por tanto, la asistencia y cuidado en el parto es una demanda prioritaria en este colectivo.

Debido a esto, no podemos olvidar que nuestra sociedad cada vez está siendo más multicultural, por lo tanto es fundamental que las enfermeras sean sensibles a las particularidades de cada cultura, ya que las diferencias en cuanto a creencias, valores y comportamientos sobre la salud determinan las expectativas de los pacientes respecto a los cuidados de enfermería. Por otra parte, las intervenciones de enfermería que incorporan prácticas específicas culturalmente adaptadas producen mayores satisfacciones en los clientes. (Amezcua, 2003).

En general uno de los mayores obstáculos con los que se encuentran todos los inmigrantes a la hora de recibir una atención sanitaria es el idioma. Debido a este problema habría que potencias recursos para su solución y de entre ellos podría contemplarse la necesidad de que en todos los servicios sanitarios se encuentre la presencia de una mediadora cultural, que conozca todos los aspectos y características de las diferentes culturas y así poder solucionar muchos de los problemas que surgen cada día.
Este nuevo sistema, que ya funciona en muchos hospitales de nuestro país, ha recibido una gran acogida por parte de los servicios médicos y de los pacientes. (www.centrodesalud.net, 2010). Otra estrategia importante para enfrentarse a la presente sociedad multicultural sería poner énfasis en la necesaria participación de enfermería junto con otros profesionales del ámbito socio-sanitario en el diseño e implementación de programas gubernamentales de atención a la población inmigrante. (Amezcua, 2003). Fundamentalmente, la función de los profesionales de enfermería debe ser de ayuda y colaboración. Debe informar, asesorar, aclarar todo tipo de dudas, como por ejemplo, las inquietudes que surgen con la decisión de usar la anestesia epidural o no. Para ofrecer cuidados culturalmente adecuados, los profesionales han de poseer conciencia cultural y sensibilidad cultural. Estas dos actitudes son vitales para conseguir una adecuada comunicación intercultural. (Pallarés, 2005).

El origen del tratamiento del dolor en el proceso del parto es tan antiguo como la humanidad (Molina, 1999). No obstante, la utilización de la analgesia epidural para este hecho se popularizó en España desde finales del siglo pasado (Torres, 1999). Para impulsar definitivamente su difusión, en España, las administraciones de las comunidades autónomas con competencias en salud han regulado su utilización, fundamentalmente durante la década de los noventa del pasado siglo (Biedma, 2010).

El grado de satisfacción es alto, un $70 \%$ muy satisfactorio. El valor más fiable es la respuesta afirmativa de todas las parturientas a repetir la analgesia epidural en un próximo parto (Campuzano, 2007). La identificación de las causas de la negativa de las mujeres inmigrantes a admitir el uso de la anestesia epidural se ha considerado como una necesidad, ya 
que el aumento de utilización de esta técnica ayudaría a la mujer a percibir el parto como algo agradable y le ayudaría a ser consciente en todo momento de la importancia de ese acontecimiento para ella misma.

Por todo lo expuesto anteriormente, en el uso de la analgesia epidural también influyen estos aspectos, es decir, por la falta de conocimientos o por la dificultad de expresarse con los profesionales sanitarios, las mujeres inmigrantes prefieren no elegir este tipo de anestesia porque creen que no van a sentir el parto igual, creen que tienen que pagar por ello, que van a tener muchas complicaciones, etc.

El objetivo de este estudio es investigar la relación entre la elección de la anestesia epidural con la nacionalidad de la mujer, y a su vez, observar si además influyen, otros factores en esta decisión. Los sujetos a estudiar son todas las mujeres que den a luz en el Hospital Comarcal del Noroeste de la Región de Murcia durante el año 2010. Se observarán unas variables y se analizarán si tienen relación con el uso de la anestesia epidural. Puede ser interesante conocer si las mujeres de otros países la utilizan menos que las españolas ya sea por motivos personales, culturales o sociales.

\section{METODOLOGÍA}

En el Noroeste de la Región de Murcia, el cual abarca cinco zonas básicas de salud (Bullas, Calasparra, Caravaca de la Cruz, Cehegín y Moratalla), había censados un total de 74.467 habitantes, en el año 2010. De éstos, 8.423 eran inmigrantes, lo que supone un $11,31 \%$ de la población. En la población inmigrante del Noroeste de la Comunidad de Murcia, en el año 2010, había 3.791 mujeres inmigrantes, de las cuáles 2.881 tenían edades comprendidas entre los 16 y 64 años, lo cual representa un $75,99 \%$ de la población. Estos datos indican que el número de partos que se producen en este municipio aumenta por la causa de embarazos de las mujeres inmigrantes. La existencia de una sociedad multicultural es una realidad creciente en nuestra comunidad (Monzón, 2006).

En el gráfico 1 se representa la muestra, es decir, el porcentaje de población del Noroeste: mujeres no inmigrantes españolas e inmigrantes que han dado a luz en el Hospital de Caravaca de la Región de Murcia. El 91,48\% (580 de 634 mujeres) corresponde a mujeres no inmigrantes españolas y el 8,51\% (54 de 634 mujeres) a mujeres inmigrantes que han dado a luz en el Hospital de Caravaca. En total 643 mujeres dieron a luz en el Hospital Comarcal del Noroeste de la Región de Murcia, pero se han excluido 9 mujeres debido a que no están registrados los antecedentes obstétricos, por lo que se quedo en una muestra de 634 mujeres.

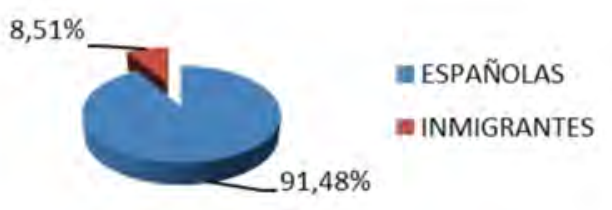

Gráfico 1: Muestra: mujeres no inmigrantes españolas e inmigrantes que han dado a luz en el Hospital de Caravaca de la Región de Murcia.

De éstas, 54 mujeres son inmigrantes, las cuales pertenecen a distintos países: 27 son sudamericanas (50\%), 2 asiáticas (3,70\%), 14 europeas $(25,92 \%)$ y 11 africanas $(20,37 \%)$. (Gráfico 2). Se ha llevado a cabo un estudio cuantitativo, transversal y descriptivo con la finalidad de conocer si existen diferencias en el uso de la anestesia epidural dependiendo de la nacionalidad, el número de abortos y la edad de la mujer. La población evaluada son 
las mujeres que han dado a luz en el Hospital Comarcal de Caravaca (Murcia) en la planta de Maternidad, durante el periodo comprendido entre el 1 de Enero al 31 de Diciembre del año 2010 ( $\mathrm{N}=634,54$ mujeres inmigrantes, las cuales representan el $8,51 \%$ del total, y 580 mujeres no inmigrantes españolas, el 91,48\%). A este hospital acuden todas las mujeres que residen en la zona del Noroeste de la Región de Murcia. El análisis estadístico de datos se ha realizado mediante el programa informático SPSS, versión 15.

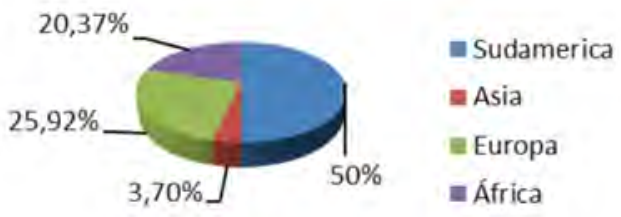

Gráfico 2: Nacionalidad de las mujeres inmigrantes

Para la descripción de las variables se ha utilizado la Chi Cuadrado de Pearson, y la tabla de contingencia. Para la realización de tablas y gráficos se ha seleccionado el programa Microsoft Excel.

El motivo por el cual las mujeres deciden no utilizar la anestesia epidural se ha representado en porcentajes.

Dicho estudio ha sido aprobado por el Comité de Ética del Hospital de Caravaca para poder utilizar los datos del programa Selene.

\section{RESULTADOS}

\section{- Proporción de mujeres}

- Mujeres que sí han utilizado la anestesia epidural: 598 (94,32\%)

- Mujeres que no han utilizado la anestesia epidural: $36(5,67 \%)$

Con una confianza del 95\%, la proporción de mujeres que piden la anestesia epidural en el Hospital Comarcal de la Región de Murcia, está comprendido entre 92,5\%; 96,1\%.

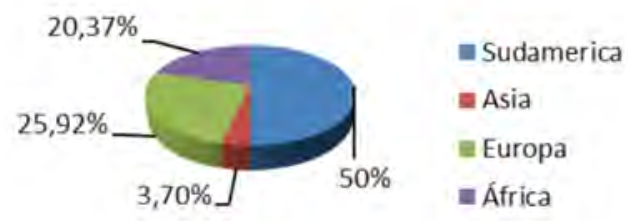

Gráfico 3: Porcentajes de la demanda de la anestesia epidural

- Nacionalidad

- Mujeres inmigrantes con epidural: 43 $(79,62 \%)$

- Mujeres inmigrantes sin epidural: 11 $(20,37 \%)$

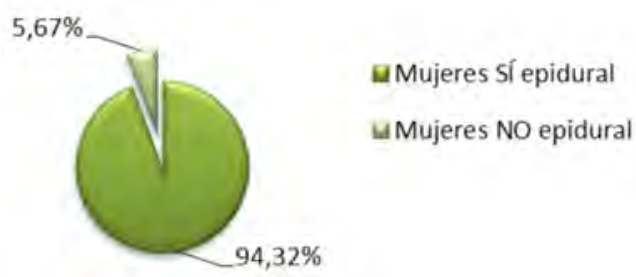

Gráfico 4: Porcentaje de mujeres inmigrantes con y sin anestesia epidural

\section{Inmigrantes}

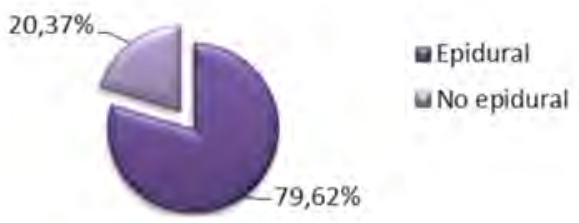

Gráfico 5: Porcentaje de mujeres no inmigrantes españolas con y sin anestesia epidural

- Mujeres españolas con epidural: 555 $(95,68 \%)$

- Mujeres españolas sin epidural: 25 $(4,31 \%)$

- Relación de la nacionalidad de la mujer con el uso de la anestesia epidural, utilizando la tabla de contingencia con un análisis de residuos y la tabla de la Chi Cuadrado. (Tabla 1) 
- Prueba chi cuadrada: X2 exp=20,886 y $\mathrm{p}<0,001$

Según los resultados obtenidos podemos afirmar que el uso de la anestesia epidural se relaciona con las mujeres no inmigrantes españolas y por lo tanto, quienes más rechazan esta anestesia son las mujeres inmigrantes.

\section{- Abortos}

- Mujeres con abortos que sí han utilizado la anestesia epidural: 117 (90,68\%)

- Mujeres con abortos que no han utilizado la anestesia epidural: $12(9,30 \%)$

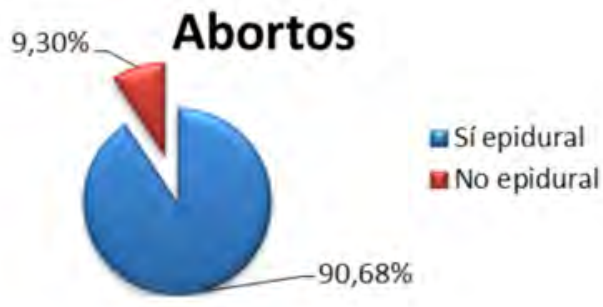

Gráfico 6: Porcentaje de mujeres con abortos, con y sin anestesia epidural

- Mujeres sin abortos que sí han utilizado la anestesia epidural: 481 (95,24\%)

- Mujeres sin abortos que no han utilizado la anestesia epidural: 24 (4,75\%)

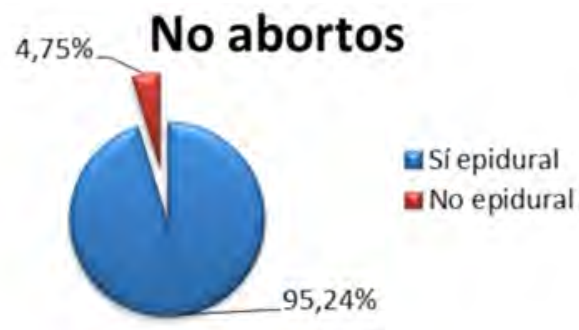

Gráfico 7: Porcentaje de mujeres sin abortos, con y sin anestesia epidural

- Relación del número de abortos de la mujer con el uso de la anestesia epidural utilizando la tabla de contingencia con un análisis de residuos y la tabla de la Chi Cuadrado. (Tabla 2)
- Chi cuadrada: X2 exp=3,972 p<0,05

Mediante estas dos pruebas, se ha determinado si el número de abortos influye en la decisión de utilizar anestesia epidural durante el parto. El resultado es que las mujeres que sí prefieren anestesia epidural son aquellas que no han tenido abortos y por lo tanto, aquellas que deciden no utilizar ningún tipo de anestesia sí han tenido algún aborto.

\section{- Edad}

- Mujeres de 16-25 años que sí han utilizado la epidural: 88 (14,71\%)

- Mujeres de 26-30 años que sí han utilizado la epidural: 130 (21,73\%)

- Mujeres de 31-35 años que sí han utilizado la epidural: 221 (36,95\%)

- Mujeres de más de 36 años que sí han utilizado la epidural: 159 (26,58\%)

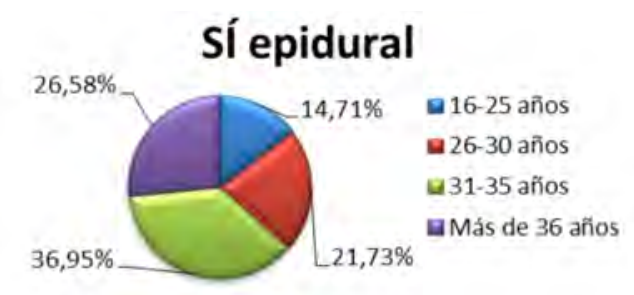

Gráfico 8: Porcentaje de mujeres con epidural, según rasgos de edad

- Mujeres de 16-25 años que no han utilizado la epidural: $11(30,55 \%)$

- Mujeres de 26-30 años que no han utilizado la epidural: $6(16,66 \%)$

- Mujeres de 31-35 años que no han utilizado la epidural: 11 (30,55\%)

- Mujeres de más de 36 años que no han utilizado la epidural: $8(22,22 \%)$

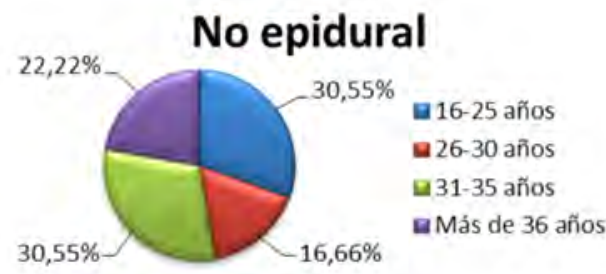

Gráfico 9: Porcentaje de mujeres sin epidural, según rasgos de edad 
- Relación edad de las mujeres y uso de la anestesia epidural, utilizando la tabla de contingencia con un análisis de residuos y la tabla de la Chi Cuadrado: (Tabla 3)

- Chi cuadrado: X2 exp=10,187 $\mathrm{p}<0,05$

Las mujeres que no prefieren utilizar anestesia epidural durante el parto son las más jóvenes, es decir, aquellas que tienen edades comprendidas entre 16-25 años. Sin embargo, las mujeres que más la solicitan durante el parto se corresponden al grupo de 31 a 35 años.

\section{- Motivos}

No se han registrado todos los motivos por los que las mujeres han rechazado la epidural pero según los datos recogidos se pueden clasificar en dos grupos:

- Parto urgente.

- No desea por motivos personales.

En este último punto deberíamos indagar más las causas así como registrarlas. En el año 2010 en el Hospital de Caravaca de la Cruz sólo se registro el 0,93\% de los motivos.

\section{DISCUSIÓN}

Durante el año 2010, el 8,51\% (N=54 mujeres) de las mujeres que dieron a luz en el Hospital de Caravaca de la Cruz eran inmigrantes. La evolución de la enfermería transcultural es necesaria ya que el 75,99\% (2881 de 3791 mujeres inmigrantes) de la población inmigrante en el año 2010 correspondía a mujeres en edad fértil.

- La nacionalidad más frecuente es Sudamérica, perteneciendo a países como: Ecuador, Bolivia, Brasil, República Dominicana, Guatemala y Colombia.

- Entre el 92,5\% y el 96,1\% ( $\mathrm{N}=598$ mujeres) de las mujeres que han dado a luz en el hospital de Caravaca de la Región de Murcia han elegido la anestesia epidural. Las mujeres inmigrantes son las que rechazan este tipo de anestesia.

- En cuanto a la nacionalidad de la mujer se ha observado que el $20,37 \%$ de las mujeres inmigrantes no han utilizado ningún tipo de anestesia habiendo una gran diferencia con las mujeres no inmigrantes españolas en las que sólo el 4,31\% no la utilizó.

- Con los resultados obtenidos se puede afirmar que existe una relación entre el uso de la anestesia epidural y el número de abortos. Aquellas mujeres que no prefieren ningún tipo de anestesia son aquellas que sí han tenido abortos. En este estudio no hay una diferencia muy significativa respecto a los abortos entre las mujeres no inmigrantes españolas e inmigrantes. Se han encontrado algunos casos más de éstas últimas mujeres (el 22,22\% se corresponde al número de abortos en mujeres inmigrantes frente al $20,17 \%$ en mujeres no inmigrantes española. Varios estudios (Manzanares, 2008; Instituto Nacional de Estadística, 2008-09; Sánchez, 2003; Marqueta, 2002), indican que las mujeres españolas tienen menos abortos que los inmigrantes debido a que estas mujeres tienen más problemas obstétricos y realizan menos consultas durante la evolución del parto que las autóctonas.

- Las mujeres que no prefieren la anestesia epidural durante el proceso del parto, según este estudio, son aquellas que están comprendidas en el rango de edad de 16 a 25 años.

- Los motivos por el que las mujeres no aceptaron la anestesia epidural no está registrada como debería, ya que sólo aparece en el $0,93 \%$ de los casos y de manera generalizada.

- Quizás los motivos de la mayoría de estas mujeres están relacionados con sus costumbres y culturas y tal vez por miedo a lo desconocido. 
- La comunicación y una mente abierta son claves para el éxito de las intervenciones en Cuidados Culturales con los pacientes. La enfermera deberá ser capaz de adaptar las intervenciones de enfermería para evitar conflictos culturales, tras la compresión del paciente de que la enfermera mantiene el respeto por su legado y la individualidad del paciente y su familia (Spector, 1999).

\section{CONCLUSIONES}

Se confirma con los resultados obtenidos que las mujeres inmigrantes hacen menos uso de la anestesia epidural. Respecto a los demás factores, las mujeres que han tenido algún aborto y aquellas con edades más jóvenes no eligen la anestesia epidural durante el parto.

Se considera fundamental la realización de Cuidados Culturales y ser sensibles hacia las diferencias cada vez mayores dada la proporción de inmigrantes estudiada. Es un reto para enfermería aprender acerca de las diferentes culturas ya que realiza su trabajo en una sociedad cada vez más multicultural.

Medidas a modo de propuesta de mejora en el cuidado a las mujeres inmigrantes:

- Reforzar la formación en enfermería transcultural.

- Reforzar el valor de los contenidos de antropología, inmigración, sociología, salud pública, cultura y cuidados de salud...en los planes de estudios.

- Aumentar la preparación y divulgación de folletos de educación sanitaria en distintas lenguas donde se explique: información sobre anticoncepción, el desarrollo normal de embarazo, consentimiento informado de analgesia epidural y en general todos los recursos sanitarios con los que se puede contar.

- Facilitar en la mujer la expresión de su forma cultural, por si es necesaria aplicarla durante el embarazo, parto o puerperio.
- Respetar y asumir aquellos conceptos y hábitos de su cultura que resulten saludables y ponerlos como ejemplo para incorporar los nuestros, de este modo se creará menor resistencia al cambio y mayor estímulo para adquirir hábitos saludables.

- Ejercer la coordinación enfermera con otras instituciones, servicios sociales, ONGS, asociaciones del propio colectivo, líderes comunitarios e imanes de las mezquitas porque nos facilitarán mucho el acercamiento y nos orientarán mejor sobre las estrategias más adecuadas para favorecer el cambio de hábitos.

- Actitud activa y participativa de los profesionales de enfermería a cualquier tipo de propuesta de cambio referido a la diversidad cultural.

\section{BIBLIOGRAFÍA}

- Amezcua. M. (2003) Conclusiones VIII Jornadas Científicas de la Fundación INDEX. "Cuidados de enfermería en una Sociedad Multicultural". Zaragoza. Rev. Índex de Enfermería. XII, 42.

- Biedma Velázquez, L; García de Diego, J.M; Serrano del Rosal, R. (2010) Análisis de la no elección de la analgesia epidural durante el trabajo del parto en las mujeres andaluzas: "la buena sufridora". Instituto de Estudios Sociales Avanzados - Consejo Superior de Investigaciones Científicas (IESA-CSIS), Junta de Andalucía, España. Rev Soc Esp Dolor; 17 (1): 3-15.

- Campuzano, C. (2007) Protocolo de analgesia epidural obstétrica en el contexto de la gestión innovadora de la asistencia y de los criterios de calidad y seguridad. Rev. Soc. Esp. Del Dolor, 14(2):117-124.

- Fernández Molina, M.A. (2006) Fenómenos migratorios y su influencia en los cuidados transculturales en el marco hospitalario. Cultura de los cuidados.10(20): 62-68.

- Manzanares Galán, S; López Gallego, F; Gómez Hernández, T; Martínez García, N; Montoya Ventoso, F. (2008) Resultados del mal control del embarazo en la población inmigrante. Prog Obstet Ginecol.; 51 (4): 215-23.

- Marqueta, J.M; Romagosa, C. (2002) Diferencias de 
morbilidad obstétrica y perinatal entre la población autóctona y magrebí. Clin Invest Gin Obst. 29 (9): 318-28. - Molina, F.J. (1999) Tratamiento del dolor en el parto. Rev Soc Esp Dolor; 6: 292-301.

- Monzón Fernández, A; Martínez Casamayor, F; Falcón Albero, A; Fernández Rodrigo, Mª.T. (2006) Estudio de las características de los partos de mujeres inmigrantes y autóctonas atendidos en el H.C.U. Lozano Blesa durante el año 2004. 1er. Premio Científico de Enfermería. Cuidando la salud. Revista científica de Enfermería. 6: 4-9.

- Pallarés Martí, A; Rifa Ros, R. (2005) Enfermería y multiculturalidad. Estrategia docente para afrontar la diversidad. Metas de Enfermería 8 (4): 16-19.
- Sánchez Fernández, Y; Muñoz-Ávalos, N; Pérez-Cuadrado, S; Robledo- Sánchez, A; Pallás-Alonso, C.R; de la Cruz-Bértolo, J. Et Al. (2003) Mujeres inmigrantes: características del control prenatal y del parto. Prog Obstet Ginecol; 46 (10): 441-7.

- Spector, R.E. (1999) Teoría y método. Cultura, cuidados de salud y Enfermería. Cultura de los cuidados:3( 6): 6679. .

- Torres, A. (1999) Más sobre la anestesia epidural obstétrica. Rev Soc Esp Dolor; 6: 403-5.

- Centro de salud.net. 4/6/2010 disponible en: http:www. centrodesalud.net/servicio-de-traduccion-en-los-hospitales/

\begin{tabular}{|c|c|c|c|c|c|}
\hline & & & \multicolumn{2}{|c|}{ Origen } & \multirow[t]{2}{*}{ Total } \\
\hline & & & Española & Inmigrante & \\
\hline \multirow[t]{2}{*}{ Epidura } & iSI & Recuento & 555 & 43 & 598 \\
\hline & NO & Recuento & 25 & 11 & 36 \\
\hline Total & & ecuento & 580 & 54 & 634 \\
\hline
\end{tabular}

Tabla 1: Tabla de contingencia complementado con un análisis de residuos

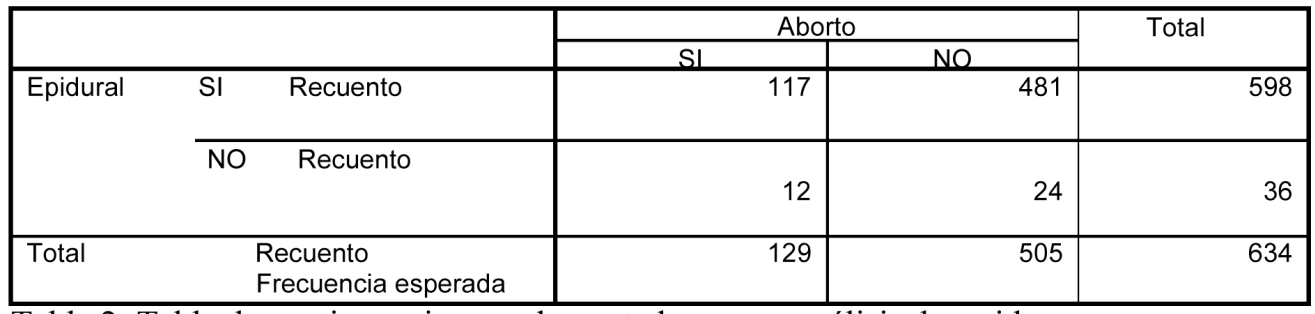

Tabla 2: Tabla de contingencia complementado con un análisis de residuos

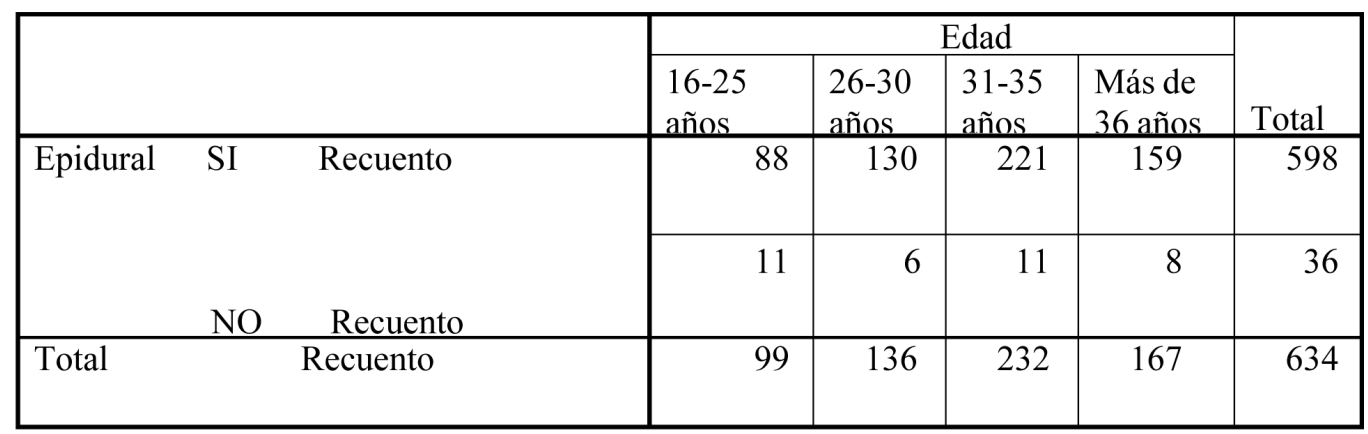

Tabla 3: tabla de contingencia complementado con un análisis de residuos 